\title{
COMPETITIVE LOCATION STRATEGIES FOR TWO FACILITIES
}

\author{
Zvi DREZNER \\ University of Michigan, Dearborn, MI 48I28, USA
}

Received November 1981, final version received March 1982

In this paper we present the problem of locating a facility when competition from another facility is taken into consideration. Two problems are addressed here. One is the location of a new facility that will attract the most buying power from an existing facility. The other is the location of a facility that will secure the most buying power against the best location of competing facility to be set up in the future.

\section{Introduction}

This paper deals with some competitive location theory on the plane. Main contributions to Nash equilibrium location problems include Hotelling (1929), Lerner and Singer (1941), Eaton and Lipsey (1975), and Wendell and McKelvey (1981). Another point of view: the Stackelberg equilibrium, was recently suggested by Hakimi (1981) for location problems on a network. In this paper we solve the Stackelberg equilibrium on the plane.

Let $n$ demand points be given on the plane, a weight, or buying power, $p_{\imath}>0$ is associated with each point; customers will buy in the new facility $Y$ if the Euclidean distance between the customer and $Y$ is less than the distance between the customer and $X$. We define the following two problems:

Problem 1. Given a location of an existing facility $X$ serving the demand points, find a location for a new facility $Y$ that will attract the most buying power of demand points.

Problem 2 (the Stackelberg equilibrium problem). Find a location for $X$ such that it will retain the most buying power against it best possible location for an additional facility $Y$. The objective function, to be minimized, for $X$ is the buying power for a best possible location of $Y$.

We can view both problems as two aspects of the same question. Let say that two facilities $X$ and $Y$ are to be located on the plane. racility $X$ has an advantage over facility $Y$ because it attracts customers in case of a tie between the distances to facility $X$ and to facility $Y$ from a demand point. Facilities $X$ and $Y$ split the available buying power in the market. Problem 1 is to find a best location for $Y$ for a given location for $X$, and Problem 2 is 


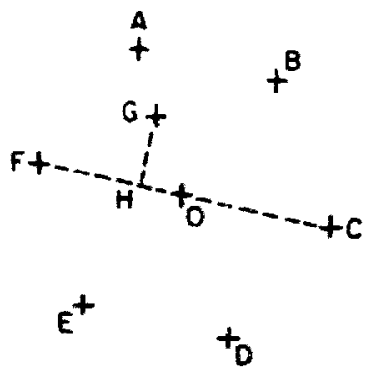

Fig 1. An example.

to find a best location for $X$ that will maximize its share of the market against the best locational strategy for $Y$.

As an example consider the problem given in fig. 1. Six demand points are located at the vertices of a sirnple hexagon with a side of length one. Equal buying power for each demand puint is assumed.

When $X$ is located at the center of the hexagon, then $Y$ can attrast three points a! most. This can be shown by the triangle inequality: The sum of distances from any point on the plane to $A$ and $D$ is at least the distance $A D$ which is equal to two. Therefore, at least one of the points $A$ or $D$ is distant at least one unit from $Y$, and thus at least one of the points $A$ or $D$ can not be attracted by $Y$ since all distances between $X$ and the demand points are equal to one unit. The same is true for the pairs $B, E$ and $C, F$. Therefore, at least three points are not covered by $Y$. It can be easily verified that three points can be attracted by $Y$ when $Y$ is located at $G$. Therefore, an optimal solution to Problem 1 when $X$ is located at the center of the polygon, has a buying power of three.

Now let us show that when $X$ is not located at the center of the hexagon, then we can always find a location for $Y$ that will attract at least four points. (Note that this location for $Y$ is not necessarily at the center of the hexagon.) To find an optimal location for $Y$ when $X$ is inside the polygon, take for example location of $X$ at $G$. The location must be off at least one of the diagonals connecting pairs of opposite points: $A D, B E$, or $C F . G$ is not on $C F$. Draw a perpendicular line from $G$ to $C F$, whose intersection point with $C F$ is $H$. If we locate $Y$ at $H$, then it must be closer than $X$ to $C$ and $F$, and it must be also closer to the other two vertices on the other side of $C F-E$ and $D$. All together $Y$ is closer to at least four points. In conclusion, the unique solution point to Problem 2 is at the center of the hexagon.

In the following sections we construct procedures for the solution of problems one and two in the general case.

\section{Solution to Problem 1}

When both locations of $X$ and $Y$ are given, then the split of the buying 
power can be found as follows. Construct the perpendicular bisector to the segment connecting $X$ and $Y$. This perpendicular bisector divides the plane into two half-planes. All points in the closed holf-plane which includes $X$ (including points on the perpendicular bisector itself) will buy at $X$. Let us call it the $X$-half-plane. All the points in the other open half-plane which includes $Y$, the $Y$-half-plane, will buy at $Y$. This leads to the following theorem:

Theorem 1. One of the optimal locations for $Y$ when $X$ is given is infinitesimally close to $X$ but not on $X$.

Proof. When $Y$ is located with $X$ the total buying power at $Y$ is zero because the distance between a customer and $Y$ is equal to the distance between the customer and $X$, and therefore all customers will buy at $X$. Therefore, no optimal solution to $Y$ is at $X$. Let $Y^{*}$ different from $X$ 's location be an optimal location for $Y$. A site $Y^{* *}$ on the open segment connecting $Y^{*}$ and $X$ is at least as good as $Y^{*}$ because the $Y$-half-plane for $Y^{* *}$ is larger than the $Y$-half-plane for $Y^{*}$. Therefore, the theorem follows. Q.E.D.

It follows from Theorem 1 that a solution to Problem 1, $Y^{*}$, is 'adjacent' (close) to $X$. The variable yet to be determined is the direction in which $Y$ is 'touching' $X$. If $Y$ is touching $X$ in a direction $A$ then the line dividing between the customers of $X$ and $Y$ is perpendicular to direction $A$ through $X$. In conciusion, finding an optimal location for $Y$ is equivalent to finding the best line through $X$ such that $Y$-half-plane defined by it contains the most buying power for $Y$.

To solve this equivalent problem we have a simple procedure: find the direction of each demand point from $X$ and sort these directions in an increasing order between 0 and $2 \pi$. Calculate in turn the buying power of all possible $Y$-half-planes which are all the points between the directions $A$ and $A+\pi$ when $A$ or $A+\pi$ is a direction to a demand point. One can easily devise the details of the algorithm. Its complexity is $O(n \log n)$ due to the sorting part, where $n$ is the number of demand points. (See also section 4 for a discussion of a more general algorithm.)

In some cases the solution for $Y$ being adjacent to $X$ is not very practical. In section 4 we extend the problem by imposing a minimal distance requirement between $Y$ and $X$.

\section{Solution to Problem 2}

While Problem 1 is quite simple, the second problem is much more interesting, i.et us first establish some properties that will help us in 
designing an algorithm for the solution of the problem. Let $f(X)$ be the maximum buying power for a new facility $Y$ when the location of $X$ is gives. $f(X)$ should be minimized in Problem 2.

Consider a line with no demand points on it. This line divides the plane into two half-planes. Let $I_{1}$ be the set of all demand points in one half-plane and $I_{2}$ be the set of all demand points in the other half-plane. Let $P_{1}$ be the buying power of the demand points in $I_{1}$, and $P_{2}$ be tise buying power of all demand points in $I_{2}$. When we choose a location for $X$ in, say, the first halfplane (which includes $I_{1}$ ) then $f(X)$, the buying power of $Y$, is at least $P_{2}$. Now, assume that there are some points on the iine. If $X$ is in the open first half-plane, then $f(X) \geqq P_{2}$. Therefore, a necessary condition for $f(X)<P_{2}$ is that $X$ belongs to the closed half-plane (including the line) which includes $I_{2}$.

Consider now a subset of demand points $I$ whose buying power is at least $P_{0}$ If $X$ is located outside the convex hull of $I$ then $Y$ can attract all members of $I$. But if $X$ belongs to the convex hull (including its boundary) then $Y$ cannot attract all the buying power of $I$. Consequently, if $X$ is inside the intersection of all convex hulls of sets with buying power greater than or equal to $P_{0}$. then $f(X)<P_{0}$. This is because $Y$ cannot attract any set whose buying power is greater than or equal to $P_{0}$.

Finally consider the set of all lines passing through pairs of demand points. There are at most $n(n-1) / 2$ such lines. Each line defines two halfplanes and each half-plane defines a set of demand points (including the points on the line itself) which consequently defines the buying power of these points. Now consider the intersection of all half-planes for which $P_{1} \geqq P_{n}$ for a given $P_{0}$. In the next theorem we show that this intersection is the same as the intersection of all conve: hulls of all sets whose buying power is at least $P_{0}$.

Theorem 2. The intersection of all contex hulls for the sets with buying power of at least $P_{0}$ is identical with the intersection of all half-planes whose huling power is at least $P_{0}$.

Prowl. Let $Z$ be a point not in the intersection of the convex hulls. Therefore. there must exist a polygon that $Z$ does not belong to it and a side of the polygon such that $Z$ is not in the half-plane defined by this side. Since the buying power of this half-plane is al least as the buying power of the set defining the polygon. the buying power of this half-plane is at least $P_{0}$ and therefore $Z$ is not in the intersection of the half-planes. Conversely, if $Z$ does not belong to the intersection of all half-planes with buying power of at least $P_{1 .}$. then there exists a half-plane that $Z$ is outside it. This half-plane is defining a set of buying power of at least $P_{0}$. which $Z$ is outside its convex hull. The theorem is proved. Q.F.I) 
Thus, $f(X)<P_{0}$ if and only it the location of $X$ beloags to the intersection of the corresponding half-planes.

Now we can present an algerithm for the solution of Probiem 2. This algorithm is basically a bisection on the buying power $f(X)$. By the above discussion it is possible to determine if there is a solution with $f(X)<P_{0}$ for some $P_{0}$ by finding if there is a feasible solution in the intersection of all half-planes whose associated buying power is at least $P_{0}$.

\section{Algorithm for Problem 2}

Step 1. Calculate all lines through pairs of points and calculate all $\boldsymbol{P}_{i}$ for each half-plane defined by the lines.

Step 2. Sort $P_{i}$ in decreasing order. Set $P_{\min }$ and $P_{\max }$ to the lowest and highest $\boldsymbol{P}_{i}$ r aspectively.

Step 3. Set $P_{0}$ to the median value in the $P_{i}$ vector for all $P_{\min }<P_{i}<P_{\max }$. If there is no $P_{i}$ fulfilling $P_{\min }<P_{i}<P_{\max }$ go to Step '7.

Step 4. Find if there is a feasible point to all half-planes for which $P_{i} \geqq P_{0}$. This can be done by linear programming.

Step 5. If there is a feasible solution point to the problem in Step 4 then $\min _{X}\{f(X)\}<P_{0}$. Set $P_{\max }$ to $P_{0}$, and go to Step 3 .

Step 6. Otherwise $\min _{X}\{f(X)\} \geqq P_{0}$. Set $P_{\min }$ to $P_{0}$, and go to Step 3 .

Step 7. A feasible point for the last $P_{\max }$ is an optimal solution. The value of the objective function is $P_{\text {min }}$.

The algorithm just described is polynomial since the linear programming part of it is polynomial by Khachian (1979). Even without the proof by Khachian we know that the procedure of finding the feasible point is polynomial because the number of variables is constant and equal to two. The number of constraints is bounded by $O\left(n^{2}\right)$ and therefore the complexity of finding a feasible point, by solving the dual problem, is bounded by $O\left(n^{4}\right)$. The complexity of the other calculations is lower, so since we perform the calculation of a feasible point $O(\log n)$ times, the complexity of the algorithm is bounded by $O\left(n^{4} \log n\right)$.

One may devise a different algorithm. Since the solution must be in the intersection of half-planes, there rnust be a feasible point which is a vertex of that intersection. Such a vertex is the intersection point of two lines. There are $O\left(n^{4}\right)$ such intersection points. For each intersection point we need to solve a Problem 1 whose complexity is $O(n \log n)$. Therefore, the complixity of this algorithm is $O\left(n^{5} \log n\right)$. We brought this algorithm up to show that the polynomiality of the the solution to Problem 2 is independent of the polynomiality of Linear Programming. 


\section{Extensiois}

4.1. One may wish, in order to be more practical, to ask for a minimal distance requirement between $Y$ and $X$ when $X$ is set up. In other words, we are not allowed to locate $Y$ within a circle of a given radius $R \geqq 0$ centered at $X$ s location. It is clear by the proof of Theorem 1 that there exists an optimal solution to Problem 1 on the circumference of that circle of radius $R$. The perpendicular bisector to a segment connecting $\dot{X}$ and a point on the circle is tangent to a circle centered at $X$ with a radius of $R / 2$. Therefore, instead of looking for a line passing through $X$ in the solution to Problem 1 , we need to look for the best $Y$-half-plane defined by a line which is tangent to a circle centered at $X$ with a radius of $R / 2$. Doing this is almost as simple as the solution proposed earlier for Problem 1 . If a demand point is inside the closed circle with a radius of $R / 2$, it will never be attracted by $Y$. For all points which are outside the circle of radius $R / 2$ we calculate two directions of tangents to this circle. If we move the tangent line around the circle, then at one direction the demand point will enter the half-plane defined by the tangent, and in another direction (not 180 apart as in the previous case) the demand point will go out of the Y-half-plane. We go over all possible Y-halfplanes and find the set with the highest buying power, which is the solution to the modified Problem 1.

Calculation of the 'enter' and 'exit' directions is done as follows. Let $d$ be the distance between a demand point and $X$. and $A$ be the direction that the demand point is seen from $X$. The enter direction is $A-\arccos (R / 2 d)$ and the exil direction is $A+\arccos (R / 2 d)$. By adding or subtracting $2 \pi$ we can get these directions to be between 0 and $2 \pi$. Let us now formulate the procedure in an algorithmic way.

\section{Algorithm for the modified Problem I}

Step 1. Calculate enter and exit directions for all demand points which are outside the circle with a radius of $R / 2$.

Step 2. Sort all enter and exit directions. In case of a tie between two directions put the exit direction first. This is because a point gets inte the $Y$-half-plane infinitesimally after the direction, but gets out of the $\gamma$-half-plane on the direction.

Step 3. Find all the points in the $Y$-half-plane for direction zero, and find the total buying power of this set.

Step 4. Check in turn all points in the sorted direction vector. If the next direction is an 'enter' add the buying power of the entering demand point to the total buying power. But if the next direction is an exit' subtract the buying power of the exiting demand point from the total buying power.

Step 5. The highest total buying power found in Step 4 is the optimum. 


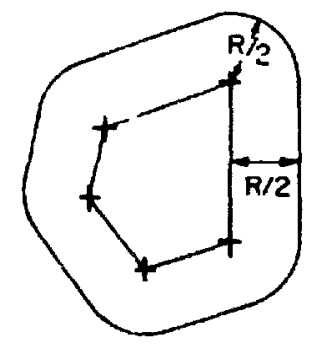

Fig. 2. The convex hull for the modified Problem 2.

Now let us turn to the modified Problem 2. Here again we do not allow the facility $Y$ to be within a distance $R$ of facility $X$. Parallel the discussion about the regular Problem 2 we see that if we have a set $I$ with buying power $P$, then we can exclude the possibility of $Y$ attracting all the demand points in the set $I$, if we locate $X$ within a distance of $R / 2$ of the convex hull of the set $I$. Unfortunately, this set in which we must locate $X$ in order :o avoid the attraction of $I$ by a possible $Y$ is not a polygon, although it is convex. (See fig. 2.)

This convex set is bounded by segments of lines distant $R / 2$ from the sides of the convex hull of the set $I$, and by arcs of circles centered at the vertices of the convex hull and with a radius of $R / 2$. It seems difficult to generalize the procedure for the regular Problem 2. However, a second approach yiclds a polynomial algorithm for this version too. The optimal solution is the intersection of the shapes of the type shown in fig. 2 for all sets for which the buying power is greater than or equal to the smallest possible $P_{0}$. If the intersection is not empty it must contain a vertex. Such a vertex is either an intersection point between two lines or between a line and a circle. or between two circles. There are $O\left(n^{2}\right)$ such lines (every pair of demand points derines two lines which are parallel to the line connecting the two points and is distant $R / 2$ from that line) and there are $n$ such circles (circles with radius $R / 2$ centered at each demand point). We have at most $O\left(n^{4}\right)$ possible intersection points between these lines or circles, as described above. For each intersection point, which is a candidate for the optimal solution, we need to solve a modified Problem 1 which is done in $O(n \log n)$. Therefore. the complexity of this algorithm is bounded by $O\left(n^{5} \log n\right)$.

4.2. Hakini (1981) has presented the problems in more general terms. Problems 1 and 2 can be formulated with $p$ facilitics of lype $X$ and $q$ facilities of type $Y$. We discussed above the case $p=q=i$. When $p=1$ and $q>1$ the problem is quite simple. The solution to Problem 1 is found as follows. When $X$ ir not located on a demand point, then there exists a line passing through $X$ with no demand points on it. Locate two $Y$ 's adjacent to 
$X$ perpendicular to that line on both sides of it. These two $Y$ 's will attrart all the buying power, and no one can do better than that. When $X$ is located on a demand point then all demand points, except the one that $X$ is located on, can be attracted by $Y$ 's as described above. Therefore, the solution to Problem 2 is to locate $X$ on the demand point with the most buying power.

When $p>1$ and $q=1$ we can solve Problem 1 with a slight modification of the problem. Let us assume that a customer will buy at $Y$ if the distance between the customer and $Y$ is less than the distance between the customer and $X$ by at least an amount of a given $\varepsilon$. The constant $\varepsilon$ can be positive, zero, or negative. Practically, the difference between the two formulations seems negligible since one can choose a very small $\varepsilon$, but the analysis is much different. For example, if $p=q=1$ and $\varepsilon=0$ then the best location for $Y$ is on $X$ attracting all demand points, compared with our formulation that yield a solution for $Y$ adjacent to $X$ and attracting only some demand points. An optimal solution procedure of complexity $O\left(n^{2} \log n\right)$ is now presented. For that, we need some additional notation. $I$ is the set of demand points. $J$ is the set of facilities of type $Y . K$ is the set of facilities of type $X . d_{i j}$ for $i \in I$, $j \in J \cup K$ is the Euclidean distance between demand point $i$ and facility $j . p_{i}$ is the buying power of demand point $i$. $\varepsilon$ is a given constant (can be negative, zero, or posit:ve).

Let

$$
R_{i}=\min _{k \in \mathrm{K}}\left\{d_{i k}\right\}-\varepsilon
$$

Definc

$$
L(Y)=\left\{i \in I \mid \min _{j \in J}\left[d_{i j}\right] \leqq R_{i j}\right\}
$$

Problem 1 is to maximize $f(Y)$, where

$$
f(Y)=\sum_{i \in L(Y)} p_{i}
$$

by the best choice of $Y$.

When $q=1$ the problem is the modified one-center problem discussed in Drezner (1981). The modified one-center problem is to find a location for a point that will cover the most buying power within given distances $R_{i}$. If $R_{i}=$ $R$ and $p_{i}=1$ then the problem is to cover the maximal number of demand points with a circle of radius $R$. The general problem (when $R_{i}$ and $p_{i}$ are not necessarily equal) for one cneter is solved there by an aigorithm of complexity of $O\left(n^{2} \log n\right)$ where $n$ is the number of demand points.

When $q>1$ then Problem 1 is a generalization of the modified one-center problem. We need to find a location for $q$ facilities of type $Y$ that will cover the most buying power within given distances $R_{i}$ to the closest facility (and if $R_{1}=R$ and $p_{i}=1$ the problem is to cover the maximal number of demand 
points with $q$ circles of radius $R$ ). This problem can be solved by the methods described in Drezner (1980). We should apply only one iteration of the optimal algorithm described there. We need to find all maximal sets and solve the resulting set covering problem, finding the maximal $F(Y)$ for all possible covers.

In conclusion, the problem yet to be solved is Problem 2 when $p>1$ and $q \geqq 1$.

\section{References}

Drezner, Z., 1980, The p-center problem $\cdots$ heuristic and optimal algorithms, ORSA/TIMS Conference, Nov. (Colorado Springs, Co).

Drezner, Z., 1981, On a modified one-center model, Management Science 27, 848-851.

Eaton, B.C. and R.G. Lipsey, 1975, The principle of minimum differentiation reconsidered: Some new developments in the theory of spatial competition, Review of Economic Studies 42, 27 . 48.

Hakimi, S.L., 1981, On locating new facilities in a competitive environment, ISOLDE 81 Conference, June (Skodsborg, Denmark).

Hotelling, H., 1929, Stability in competition, Economic Journal 39, 41-57.

Khachian, L.G., 1979, A polynomial algorithm in linear programming (English translation). Soviet Mathematics Doklady 20, 191-194.

Lerner, A. and H. Singer, 1941, Some notes on duopoly and spatial competition, Journal of Political Economy 45, 423-439.

Wendell, R.E. and R.D. McKelvey, 1981. New perspectives in competitive location theory. European Journal of Operational Research 6, 174-182. 